II Congresso Brasileiro de

Fluidodinâmica Computacional

27 a 29 de Junho de 2018

Rio de Janeiro, RJ

\title{
PARAMETRISATION OF GEOMETRY VIA COLLISION ALGORITHM IN THE FRAMEWORK OF FULL NAVIER - STOKES EQUATIONS FOR TURBULENT FLOWS
}

\author{
T. D. FERREIRA ${ }^{1}$ AND S. S. V. VIANNA ${ }^{1}$ \\ ${ }^{1}$ University of Campinas, School of Chemical Engineering \\ Email para contato: tatieledf@feq.unicamp.br / savio@feq.unicamp.br
}

\begin{abstract}
The parametrisation of geometrical stereolithography CAD files is investigated within the discretisation of the full Navier-Stokes equations. The geometry is blended into hexa shaped mesh via a collision approach of convex sets. All parts that comprise the geometry are treated individually and the finite volumes in the mesh are tagged based on the collision between the geometry and the mesh. The volume and areas of the computational cells are amended following a pre-stablished porosity value. The turbulent flow is solved in the mesh using RANS (Reynolds Averaged Navier Stokes) approach and the turbulence problem is closed by the Boussinesq formulation solving two additional equations for turbulent kinetic energy and its respective rate of dissipation. Comparison with experimental data for the classical step case presented good agreement.
\end{abstract}

\section{INTRODUCTION}

Natural phenomena including those with motion of fluids are of interest of many scientific studies. One of the main subjects from the area of fluid dynamics is the study of turbulent flows. The computational fluid dynamic (CFD) technique is one of the available approaches to model turbulent fluid flow. In traditional CFD codes a CAD file is taken as geometry of interest and the computational mesh is built in the computational domain. The main drawback of such technique is the representation of all geometrical scales: for small objects, a very fine mesh is required which greatly increase the computational costs.

This work investigates how the collision algorithm can be considered in the framework RANS (Reynolds Navier-Stokes) formulation. The numerical results are compared with experimental data for the turbulent flow over the backward facing step and good agreement is observed.

\section{PARAMETRISATION VIA COLLISION ALGORITHM}

The Gilbert-Johnson-Keerthi Distance algorithm (GJK algorithm), (Gilbert et. al, 1989) (Gilbert et. al, 1988) is a method for obtaining the minimum distance between two convex objects based on the concept of the Minkowski difference. This algorithm can also be used to check for collision between two convex objects.

The main advantage of the GJK algorithm is the stability and speed. The algorithm also relies on a support function that makes it possible to check for collisions between any kind of discrete and analytical objects, in any kind of format. 


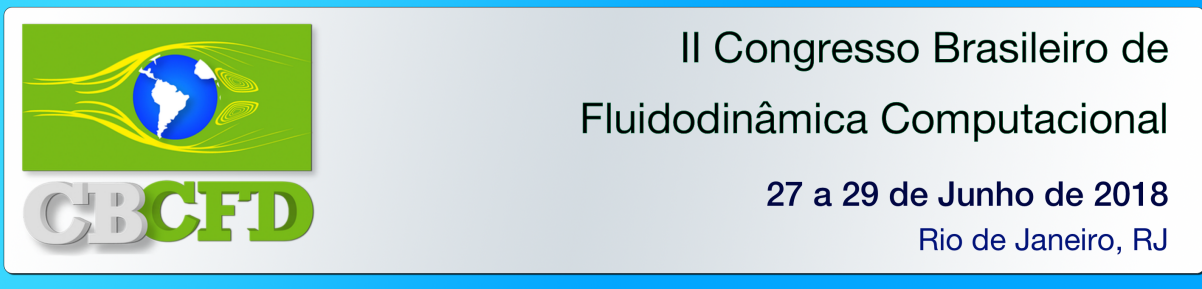

In order to parametrise a geometry in a computational mesh for CFD calculations, the GJK algorithm is applied to check for a collision between an element of the hexahedral mesh and each solid of the geometry. This process is described in Figure 1: on step 1 a geometry is imported into a domain; on step 2 a structured mesh is created over the domain; on step 3 the GJK technique is used to calculate the porosity of the mesh.
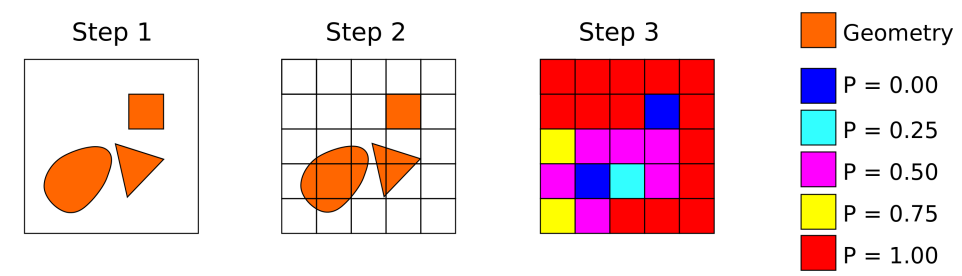

Figure 1 - Steps of the porosity generation.

In this approach, every mesh element fully occupied by a solid has a porosity value of 0.0, while every empty element has a porosity value of 1.0. The porosity is proportional, so if an element of the mesh is half full its value is 0.5 .

The final parametrised geometry presents values of porosity applied to the volume and faces of each computational cell. Such values are coupled into the differential equations that govern the fluid flow.

\section{MODIFIED TRANSPORT EQUATIONS APPLIED TO A POROUS MESH}

The governing equations for turbulent flow presented in this work are modified to take into account the porosity values related to each computational cell. The porosity values for the area $\left(\beta_{j}\right)$ and volume $\left(\beta_{V}\right)$ are computed according to the blockage of the computational cell throughout the GJK algorithm.

Using a generic variable $\phi$, the quantities: mass, momentum, energy, turbulent kinetic energy and eddy dissipation rate; are expressed as:

$$
\int_{V} \frac{\partial(\rho \phi)}{\partial t} \beta_{V} d V=-\int_{S}\left(\rho u_{j} \phi\right) \beta_{j} d S_{j}+\int_{S} \Gamma_{\phi} \frac{\partial \phi}{\partial x_{j}} \beta_{j} d S_{j}+\int_{V} S_{\phi} \beta_{j} d V
$$

The values of $\beta_{V}$ and $\beta_{j}$ ranging from 0.0 (when the cell is fully blocked) to 1.0 (when the computational cell is completely open).

The governing equations in format of equation 1 are solved for each quantity in an in house Navier-Stokes solver named PFS (Porosity Flow Solver). The solver comprises an explicit code based on the finite volume method. The central difference scheme is applied to discretise the governing equations and a fourth order Runge-Kutta algorithm is applied for time-marching. Artificial viscosity is added to dump the wiggling behaviour of the numerical scheme and solution decoupling. 


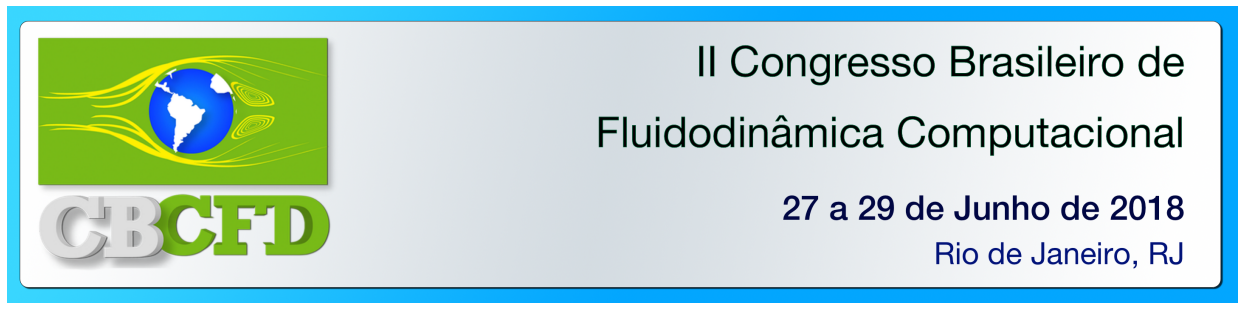

\section{RESULTS}

A case study of a turbulent flow using a porous mesh obtained by the GJK algorithm to model the geometry under study is discussed here.

Based on previous experimental study (Kim et. al, 1978) and numerical simulation (Yoo et. al, 1989), a fluid flow at Reynolds number equal to 44,000 was simulated over a step with $10 \mathrm{~mm}$ of height (h) and $40 \mathrm{~mm}$ of length. The computational domain used in this case comprises a rectangular box with $340 \mathrm{~mm}$ in $\mathrm{x}$ direction and $3 \mathrm{~mm}$ in $\mathrm{z}$ direction e $10 \mathrm{~mm}$ in y direction and computational cells with $1 \mathrm{~mm}$ of length were considered. Figure 2 shows the porosity values calculated for each computational cell. Here, the blue region represents the step geometry (computational cells were set to zero porosity), while the red region represents available domain to the flow field calculation.

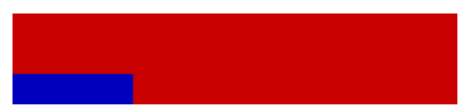

Figure 2 - Computational domain used in the study of a turbulent flow over a step.

Different types of boundary conditions were considered. The flow entrance in the computational domain was considered at the left boundary. The total pressure, temperature, turbulent kinetic energy and dissipation and velocity are specified. Based on the considered Reynolds number $(44,000)$, an air flow with velocity of $70 \mathrm{~m} / \mathrm{s}\left(U_{\infty}\right)$ was considered. The outlet boundary condition, with pressure gradient equal to zero, was considered at the right boundary. The other borders of the computational domain were considered as wall where zero normal fluxes of mass, momentum and energy are imposed. Figure 3 presents the result of the simulation. In the inner picture it is possible to observe the presence of a re-circulation zone downstream the step. The local mean velocity profiles and turbulence intensity at two different flow regions downstream the step are also observed. The results are compared against experimental data and the simulation conducted by Yoo, 1989. The velocity profiles and turbulence intensity provided by PFS code are very similar to the experimental data at the re-circulation region which indicates that proposed model reproduced the velocity behaviour at the separation zone of a turbulent flow over a step.

An important factor is to analyse the reattachment length. Experimental study of Kim et. al presents a mean reattachment distance of $x / h=7 \pm 1$ for turbulent flow over backward-facing step. The simulation provides a reattachment length of $x / h=8.5$ which is very close to the expected value according to experimental study.

\section{CLOSING REMARKS}

The Gilbert-Johnson-Keerthi Distance algorithm (GJK algorithm) has been proved as good alternative for parametrisation of geometries as porous media in CFD studies. The porosity values provided by GJK algorithm were coupled in a Navier-Stokes solver and good agreements with experimental data were finding for the simulation of turbulent flow over a step. 

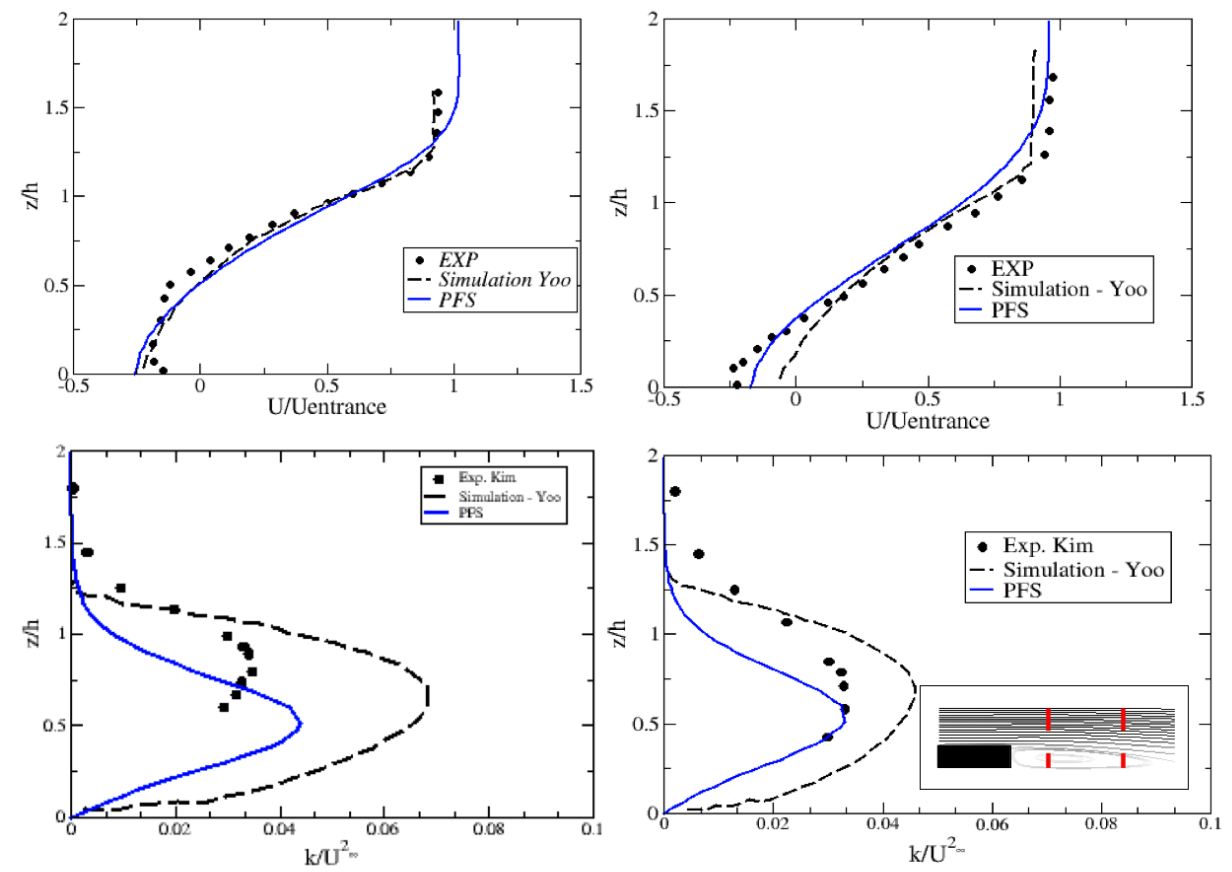

Figure 3 - Numerical findings for the step case and associated streamlines (inner picture) are presented. Upper plots report the normalised velocity while lower plots report the turbulence intensity. Both results were taken along the vertical line (red line in the inner plot) at two positions downstream the step.

\section{ACKNOWLEDGEMENTS}

Thanks are due to CNPq, University of Campinas and L4R1S4 laboratory.

\section{REFERENCES}

GILBERT, E.G.; JOHNSON, D.W.; KEERTHI, S.S. A fast procedure for computing the distance between complex objects in three-dimensional space. IEEE Journal of Robotics and Automation, 2, 193-203, 1988.

GILBERT, E.G.; FOO, C.P.Computing the distance between smooth objects in three dimensional space. IEEE International Conference on Robotics and Automation, 1989. Proceedings., 1989, 1, 158-163, 1989.

KIM, J.; KLINE, S. J.; JOHNSTON, J. P.Investigation of separation and reattachment of a turbulent shear layer: flow over a backward-facing step. Thermosciences Division, Dpto of Mechanical Engineering, Stanfor University, 1978.

YOO, J.Y.; CHOI, H.C..; HAN, S.M. Numerical analysis of turbulen flow over a backward-facing step using Reynolds Stress closure model. IEEE Journal of KSME, 1, 31-37, 1989. 